\title{
Kondo Lattice without Nozieres Exhaustion Effect
}

\author{
K. Kikoin ${ }^{1}$ and M.N. Kiselev ${ }^{2}$ \\ ${ }^{1}$ Ben-Gurion University of the Negev, Beer-Sheva 84105, Israel \\ ${ }^{2}$ Physics Department, Arnold Sommerfeld Center for Theoretical Physics and Center for Nano-Science, \\ Ludwig-Maximilians Universität München, 80333 München, Germany
}

(Dated: December 7, 2018)

\begin{abstract}
We discuss the properties of layered Anderson/Kondo lattices with metallic electrons confined in $2 \mathrm{D} x y$ planes and local spins in insulating layers forming chains in $z$ direction. Each spin in this model possesses its own 2D Kondo cloud, so that the Nozieres' exhaustion problem does not occur. The excitation spectrum of the model is gapless both in charge and spin sectors. The disordered phases and possible experimental realizations of the model are briefly discussed.
\end{abstract}

PACS numbers: 71.27.+a,75.20.Hr,75.10.Pq,75.30.Mb

Famous exhaustion problem formulated by Nozieres [1] is a stumbling stone on the way from exactly solvable Anderson or Kondo impurity model 2] to the periodic 3D Anderson lattice (AL) or Kondo lattice (KL) models, which are believed to provide the generic Hamiltonians for mixed valence and heavy fermion materials 3,4$]$. The problem arises already for concentrated Kondo alloys where the number or localized spins $N_{i}$ is comparable with the number of sites $N=L^{n}$ in the $n$-dimensional lattice. In this case the number of spin degrees of freedom provided by conduction electrons in a $\mathrm{KL}$ is not enough for screening $N_{i}$ localized spins. As an option a scenario of dynamical screening was proposed, [5, 6] where only part of spins screened by Kondo clouds form magnetically inert singlets. The low-temperature state of such KL is a quantum liquid, where $N_{s}$ singlets are mixed with $N-N_{s}$ "bachelor" spins, which hop around and exchange with singlets thereby behaving as effective fermions. The Nozieres' exhaustion is measured by a parameter $p_{N}=N_{i} / \rho_{0} T_{K}$ (the number of spins per screening electron). Here $T_{K} \sim \rho_{0}^{-1} \exp \left(-1 / \rho_{0} J\right)$ is the energy scale of Kondo effect, $J$ is the exchange coupling constant in the single-impurity Kondo Hamiltonian, $\rho_{0}$ is the density of states on the Fermi level of metallic reservoir.

Second obstacle, which does not allow the extrapolation of Kondo impurity scenario to KL is the indirect RKKY exchange $I_{j j^{\prime}}$ between the localized spins, which arises in the 2nd order in $J$ or in the 4th order in $V$ (hybridization parameter in the generic AL Hamiltonian). The corresponding energy scale is

$$
\rho_{0} I=\rho_{0} J^{2} \chi_{j j^{\prime}}^{c} \sim\left(\rho_{0} J\right)^{2},
$$

where $\chi_{j j^{\prime}}^{c}=N^{-1} \sum_{\mathbf{q}} \chi_{c}(\mathbf{q}) \exp \left(i \mathbf{q} \cdot \mathbf{R}_{j j^{\prime}}\right)$ and $\chi_{c}(\mathbf{q})$ is the spin susceptibility of the electron gas. The Fourier transform of $\chi_{c}(\mathbf{q})$ is an oscillating function, which strongly depends on the distance $R_{j j^{\prime}}$. If $I<0$ at an average inter-impurity distance, and $|I| \sim T_{K}$, then the trend to inter-site antiferromagnetic (AF) coupling competes with the trend to the one-site Kondo singlet formation (Doniach's dichotomy [] $]$ ).

This competition prompted several possibilities to by- pass the exhaustion limitations. According to a scenario offered in $\left[8,[9]\right.$, in the critical region $|I| \sim T_{K}$ of Doniach's phase diagram, where the AF correlations are nearly suppressed by the on-site Kondo coupling, the spin liquid phase enters the game. This phase is characterized by the energy scale

$$
\rho_{0} \mathcal{I}(T)=\rho_{0} J^{2} \chi_{j j^{\prime}}^{s}(T)
$$

where $\chi_{j j^{\prime}}^{s}$ is the spinon susceptibility. The condition $\mathcal{J}\left(T_{K}\right)>T_{K}$ is easily achieved both in $3 \mathrm{D}$ and $2 \mathrm{D}$ case. The Kondo screening is then quenched in the weak coupling regime at $T>T_{K}$, so that the spin degrees of freedom remain decoupled from the electron Fermi-liquid excitations both at high temperatures $T \gg T_{K}$ and at low temperatures $T \ll T_{K}$ (Curie and Pauli limit for magnetic response, respectively). At $T \rightarrow 0$ the KL behaves as a two-component Fermi liquid with strongly interacting charged electrons and neutral spinons [10]. This scenario develops on the background of strong AF correlation. It includes the possibility of ordered magnetic phases with nearly screened magnetic moments and, in particular, the quantum phase transitions. Due to separation of spin and electron degrees of freedom, the Luttinger's theorem in its conventional Fermi-liquid form is invalid in this state: $f$-electrons represented by their spin degrees of freedom give no contribution in formation of the electron Fermi surface. Such state is referred as a "small Fermi surface regime" in current literature.

Another scenario for small Fermi surface regime was proposed in 11]. This scenario appeals to systems where the magnetic order is either fragile or entirely absent due to magnetic frustrations (e.g., to triangular lattices). A spinon gap carrying unit flux of $Z_{2}$ gauge field is expected to arise in spin subsystem, and this gap prevents formation of Kondo singlets for a finite range of $T_{K}$. As a result the Nozieres' exhaustion does not occur, and fractionalization of excitations into spin-fermions and electrons exists like in the previous case. A possibility of forming the spin liquid with $U(1)$ gauge group and spin density wave ground state has also been pointed out in 11].

In the present paper we propose another paradigm for 

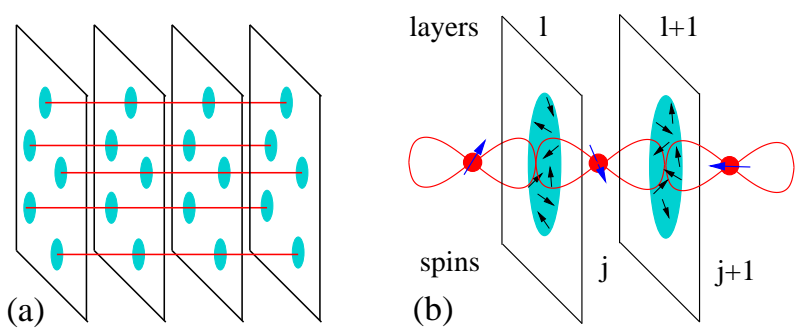

FIG. 1: (a) Layered lattice of spatially separated charges in planes and spins in chains. (b) A fragment of a chain with Kondo clouds formed as "shadows" in metallic layers.

fermion fractionalization in Kondo lattices, which possesses the generic properties of KL but is not subject to the exhaustion limitations. This paradigm may be realized in strongly anisotropic Kondo lattices, where the metallic electrons are confined in 2D planes interlaid by insulating layers containing magnetic ions. Then the 3D reservoir of screening electrons is defragmented into $L$ planar reservoirs. Each plane still possesses the macroscopic number of spin degrees of freedom $\sim L^{2}$ enough for Kondo screening, provided the concentration of magnetic centers per metallic plane remains small. The spin liquid features may be observed in these systems if the distribution of magnetic centers is also anisotropic, namely, if they form chains oriented in $z$ direction, and the interchain interaction is negligibly small.

Leaving the discussion of experimental realization of such systems for concluding section, we begin with the theoretical description of an ideal configuration, where all chains penetrate the stack in $z$ direction (Fig पi ). The AL Hamiltonian for the quasiperiodic model of conduction electrons confined in metallic layers ( $x y$ plane), and magnetic ions localized in insulating layers between metallic planes is

$$
\begin{aligned}
H & =\sum_{l \mathbf{k} \sigma} \epsilon_{k} c_{l \mathbf{k} \sigma}^{\dagger} c_{l \mathbf{k} \sigma}+\sum_{j \sigma}\left(\epsilon_{d} n_{j \sigma}^{d}+\frac{1}{2} U n_{j \sigma}^{d} n_{j \bar{\sigma}}^{d}\right) \\
& +V \sum_{j l} \sum_{\mathbf{k} \sigma}\left(c_{l \mathbf{k} \sigma}^{\dagger}\left(d_{j \sigma}+d_{j+1, \sigma}\right)+h . c\right)
\end{aligned}
$$

Here $\mathbf{k}$ is a $2 \mathrm{D}$ wave vector, the discrete indices numerate metallic layers $l$ with a lattice constant $a_{\|}$and magnetic sites $j$ along the chains with a spacing $a_{z}$. The coupling constant $V$ characterizes hybridization between itinerant $2 \mathrm{D}$ electrons in a plane $l$ and localized states in two adjacent chains $j$, and $j+1$ (Fig.1b). We treat the electrons in metallic planes in terms of Bloch waves $c_{l \mathbf{k} \sigma}$, while the localized electrons are characterized by Wannier functions $d_{j \sigma}$. The lattice is quasiperiodic in a sense that the periodicity of magnetic sites in the $x y$ plane is not demanded, but the average distance $\lambda$ between the impurities within a layer exceeds the radius of Kondo cloud, i.e. satisfies the condition $\lambda \ll \hbar v_{F} / T_{K}\left(v_{F}\right.$ and $T_{K}$ are Fermi velocity of $2 \mathrm{D}$ electrons and Kondo temperature, respectively). There is no interaction between the chains under this condition, and a single chain represents the $z$ component of excitation spectrum. On the other hand, all chains contribute to the $x y$-component of the spin and charge response of the AL. The effects associated with the inter-chain exchange will be discussed in the concluding part.

We came to a situation where $L$ two-dimensional Fermi reservoirs, each with capacity $L^{2}$, screen $N_{i}$ magnetic moments arranged in such a way that the effective concentration of these moments per metallic layer is $n_{i}=N_{i} / L^{2}$ satisfies a condition $n_{i} a_{\|}^{2} \ll 1$. This capacity is enough to form screening Kondo cloud for each magnetic site within a given layer $l$ independently of all other sites belonging to the same layer. On the other hand, two magnetic ions localized one above another in neighboring insulating layers $j, j+1$ share the same metallic screen (see Fig. 1b). Replicating these dimers along $z$ axis, one we come to a system of spin chains, interacting with a system of metallic layers stacked up in the $x y$ plane. Elimination of the hybridization term $V$ in the Hamiltonian (3) in accordance with the standard Schrieffer-Wolff procedure, results in effective exchange Hamiltonian for each chain,

$$
H_{i n t}^{c d}=\sum_{j=1, k, k^{\prime}}^{N_{i}} J_{\mathbf{k k}^{\prime}} \vec{s}_{l+1, \mathbf{k} \mathbf{k}^{\prime}}\left(\vec{S}_{j}+\vec{S}_{j+1}\right)
$$

(see Fig. 10). Here $\vec{s}_{l+1, \mathbf{k} \mathbf{k}^{\prime}}=\frac{1}{2} c_{l+1, \mathbf{k} \sigma}^{\dagger} \vec{\sigma}_{\sigma \sigma^{\prime}} c_{l+1, \mathbf{k}^{\prime} \sigma^{\prime}}$, $\vec{S}_{j}=\frac{1}{2} d_{j, \sigma}^{\dagger} \vec{\sigma}_{\sigma \sigma^{\prime}} d_{j, \sigma^{\prime}}$, and an exchange integral is estimated as $J \sim V_{\mathbf{k}}^{*} V_{\mathbf{k}^{\prime}} / U$.

Thus, the original model is reduced to the anisotropic KL formed by the system of $1 \mathrm{D}$ spin chains penetrating the stack of 2D metallic layers. Each spin creates two Kondo clouds in adjacent planes, and two neighboring spins see each other through a metallic screen by means of indirect RKKY-like exchange. This exchange may be either ferromagnetic or antiferromagnetic. In this work the latter case is considered in terms of the Doniach's dichotomy [7]. In our model this dichotomy should be reformulated. Since the long-range AF ordering is impossible in 1D chain, two competing phases are Kondo singlet and spin liquid. Complete Kondo screening is not forbidden by Nozieres' exhaustion principle, since the 2D screening layer is available for each spin in the chain. The Kondo screening is characterized by the energy scale $T_{K}$. Thus, the competing phases in the anisotropic KL are the Kondo singlet phase and the homogeneous spin liquid of RVB type with the energy scale given by Eq. (2).

In order to describe the Doniach-like phase diagram we adopt the method of [9]. Namely, we derive an effective action functional by integrating out all "fast" fermionic degrees of freedom with the energies $\sim D_{0}$, where $2 D_{0}$ is the conduction bandwidth. The "slow" modes give us a hydrodynamic action. Due to strong quasi 1D anisotropy 
there is no need in appealing to the mean-field approximation. After elimination of conduction electrons with the energies $D_{0}>\varepsilon>T$ in metallic layers, the coupling $J$ is enhanced, $J \rightarrow \tilde{J}=1 / \rho_{0} \ln T / T_{K}$. The indirect RKKY-like spin-spin interaction mediated by the in-plane electrons arises along the chains:

$$
H_{i n t}^{d d}=-I \sum_{j, \sigma \sigma^{\prime}} d_{j \sigma}^{\dagger} d_{j+1, \sigma} d_{j+1, \sigma^{\prime}}^{\dagger} d_{j \sigma^{\prime}}
$$

Here $I$ is defined in Eq. (11) with $\chi_{j, j+1}^{c}=$ $N^{-1} \sum_{\mathbf{q}} \chi_{c}(\mathbf{q}) \exp i\left(q_{z} a_{z}\right)$. Since $V / U \ll 1$, we adopt the nearest-neighbor approximation for RKKY interaction.

Up to this moment we treated the spin chains in a single-site approximation. This approximation is legitimate until $T \gg T_{K} \sim I$. To move further, we decouple the Euclidean action of the model (4), (5)

$$
\mathcal{A}=\int_{0}^{\beta} d \tau\left[\sum_{j}\left(\bar{c} \mathcal{G}_{0}^{-1} c+\bar{d} \mathcal{D}_{0}^{-1} d\right)-H_{\text {int }}^{c d}-H_{\text {int }}^{d d}\right]
$$

by means of the Hubbard-Stratonovich scheme [12] in terms of the fields $\Delta_{j, j \pm 1} \rightarrow \sum_{\sigma}\left(d_{j \sigma}^{\dagger} d_{l \pm 1, \sigma}+c . c\right)$, $\phi_{l} \rightarrow \sum_{\mathbf{k} \sigma}\left(c_{l-1, \mathbf{k} \sigma}^{\dagger}\left(d_{j, \sigma}+d_{j+1, \sigma}\right)+c . c\right)$ [9, 13]. Here $\mathcal{G}_{0}^{-1}=\partial_{\tau}-\epsilon(-i \nabla)+\mu$ and $\mathcal{D}_{l o c}^{-1}=\partial_{\tau}-i \pi /(2 \beta)$ are bare inverse single particle Green's Functions (GF) for conduction electrons and local spins, respectively, $\beta=1 / T$. The field $\phi$ describes the single-site Kondo screening and the field $\Delta$ stands for the spinon propagation along the 1D spin chain with AFM coupling. The single occupancy constraint $d_{j \uparrow}^{\dagger} d_{j \uparrow}+d_{j \downarrow}^{\dagger} d_{j \downarrow}=1$ is preserved at each site in the chain by the semi-fermionic transformation 14]. These two fields resolve the Doniach's dichotomy, because the long-range AF order is absent in 1D.

We appeal to the uniform resonance valence bond (RVB) spin liquid state [13] and treat the spinon modes as fluctuations around the homogeneous solution in a $n n$ approximation, $\Delta_{j, j \pm 1} \rightarrow=\Delta_{j, j \pm 1}^{u}-\bar{\Delta}$ with

$$
\bar{\Delta}^{2}(\beta)=\beta^{-1} \int_{0}^{\beta} \Delta(\tau) \Delta(-\tau) d \tau .
$$

For this sake we add and subtract $\bar{\Delta}$ in the inverse GF. The non-local inverse spinon GF $\mathcal{D}^{-1}=\partial_{\tau}-$ $\Delta_{j, j \pm 1}-i \pi T / 2$. has to be expanded in terms of $\Delta-$ $\bar{\Delta}$. Now the two interacting components of bose-like modes in two-sublattice chain are spinons and Kondo clouds represented in effective action by $\Delta_{j, j+1} \Delta_{j+1, j}$ and $\phi_{l, l+m} \phi_{l+m, l}(m=0,1)$, respectively. The charged $\phi$-mode acquires dispersion due to the non-locality of $\tilde{J}_{l j}$, while the in-plane dispersion of conduction electrons in Kondo clouds is integrated out. The neutral spinon mode
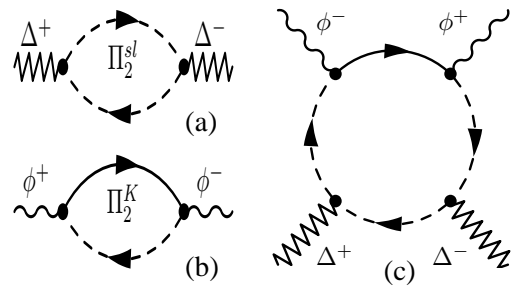

FIG. 2: Loop expansion for non-local action (7) Solid and dashed lines in $\Pi_{2}$ and $\Pi_{4}$ stand for electron and spinon propagators, respectively.

is dispersive by its origin. The action in these terms is

$$
\begin{aligned}
& \mathcal{A}_{\text {eff }}=\sum_{j l, \omega_{n}}\left(\frac{\left|\phi_{l, l}\left(\omega_{n}\right)\right|^{2}}{\tilde{J}_{l j}}+\frac{\left|\Delta_{j, j+1}\left(\omega_{n}\right)\right|^{2}}{I}\right)+ \\
& \operatorname{Tr} \log \left(\mathcal{G}_{0}^{-1}\right)+\operatorname{Tr} \log \left(\mathcal{D}^{-1}\left(\Delta_{j, j+1}\right)+\mathcal{G}_{0} \phi_{l, l}^{*} \phi_{l, l \pm 1}+\text { c.c }\right)
\end{aligned}
$$

As usual in 1D systems, the spin and charge sectors in $\mathcal{A}_{\text {eff }}$ are separated.

The last term in (7) may be represented as a loop expansion. Two first diagrams are shown in Fig.2. To calculate the diagrams, we use the non-local spinon GF $\mathcal{D}_{j, j+r}^{0}\left(\omega_{n}\right)=\left(\mathcal{D}_{l o c}-\bar{\Delta}\right)^{-1}$ with cosine-like dispersion

$$
\mathcal{D}_{j, j+r}^{0}\left(\omega_{n}\right)=\frac{\exp \left(-|r|\left[\ln \left(\frac{\omega_{n}-\sqrt{\omega_{n}^{2}+\bar{\Delta}^{2}}}{\Delta}\right)+i \frac{\pi}{2}\right]\right)}{i \sqrt{\omega_{n}^{2}+\bar{\Delta}^{2}}} .
$$

Here $r$ numerates sites in the chain, $\omega_{n}=2 \pi T(n+1 / 4)$ on the imaginary axis 14. $\mathcal{D}^{0}$ is characterized by two branch cuts at $[\bar{\Delta},+\infty)$ and $(-\infty,-\bar{\Delta}]$. In the limit $\bar{\Delta} \ll \pi T$ it rapidly falls down with growing $|r|$ as $\mathcal{D}_{j, j+r}^{0}\left(\omega_{n}\right) \sim-\bar{\Delta}^{|r|} /\left(-i \omega_{n}\right)^{|r|+1}$. Thus the main contribution comes from $\mathcal{D}_{j, j}^{0}\left(\omega_{n}\right)=-i / \sqrt{\omega_{n}^{2}+\bar{\Delta}^{2}}$ and $\mathcal{D}_{j, j \pm 1}^{0}\left(\omega_{n}\right)=\left(\omega_{n} / \sqrt{\omega_{n}^{2}+\bar{\Delta}^{2}}-1\right) / \bar{\Delta}$.

The polynomial effective action after the loop expansion is performed is given by

$$
\begin{aligned}
& \mathcal{A}_{e f f}=\sum_{\left\langle j j^{\prime}\right\rangle \omega_{n}}\left[\frac{\left|\Delta_{j j^{\prime}}\left(\omega_{n}\right)\right|^{2}}{I}-\Pi_{2}^{s l}\left|\Delta_{j j^{\prime}}\left(\omega_{n}\right)-\bar{\Delta}\right|^{2}\right] \\
& +\sum_{j j^{\prime} l, \omega_{n}}\left(\frac{1}{\tilde{J}_{j l}}-\Pi_{2}^{K}+\Pi_{4}^{K}\left|\Delta_{j j^{\prime}}\left(\omega_{n}\right)\right|^{2}\right)\left|\phi_{l j}\left(\omega_{n}\right)\right|^{2} \\
& +\operatorname{Tr} \log \left(\mathcal{G}_{0}^{-1}\right)+\operatorname{Tr} \log \left[\left(\mathcal{D}^{0}\right)^{-1}\right]+O\left(|\phi|^{4}\right) .
\end{aligned}
$$

The polarization loops $\Pi_{2}$ and $\Pi_{4}$ are shown in Fig.2. The action (8) is gauge invariant in accordance with Elitzur theorem [13], and spin and charge modes are separated both in the 3D lattice and in the Fock space.

To estimate $\bar{\Delta}$, we refer to the properties of spin chains with AF coupling 15. The quasi-long-range-order in these chains may be treated in terms of boson excitations in Luttinger liquid (LL) or fermion pairs in spin liquid. 

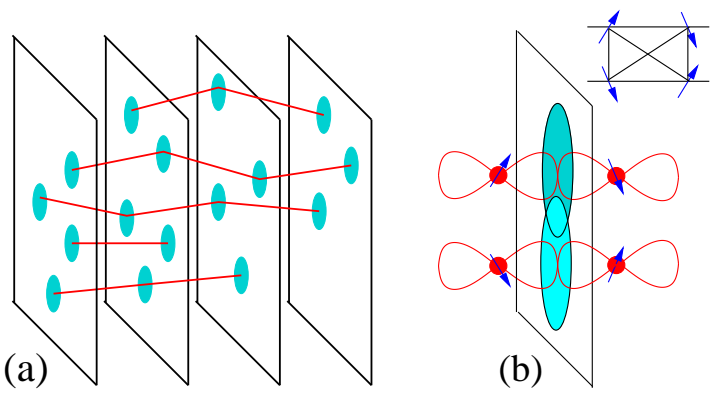

FIG. 3: (a) Disordered anisotropic Kondo lattices ; (b) Formation of spin ladder from interacting chains.

The spin susceptibility of a chain, $\left\langle\Delta^{+} \Delta^{-}\right\rangle_{\omega=0} \sim \bar{\Delta}^{2}$, acquires Pauli form at $T^{*} \sim 8 J^{2} / E_{F}$ [16], so we assume $\bar{\Delta} \sim T^{*}$ in our estimates. This means that even in the critical region of Doniach's diagram, $T_{K} \approx \tilde{J}^{2} / E_{F}$, the spins are "molten" into spin liquid at $T \sim T_{K}$, and the Kondo screening is irrelevant at low $T$.

Evaluation of $\Pi_{2}, \Pi_{4}$ in the limit $\pi T \gg \bar{\Delta}$ gives $\Pi_{2}^{K} \sim \rho_{0} \ln (\bar{\Delta} / T)$ and $\Pi_{4}^{K} \sim \rho_{0} / \bar{\Delta}^{2}$. This leads to reduction of indirect exchange, $\tilde{I}=I\left[1+I / \bar{\Delta} \ln \left(\bar{\Delta} / T_{K}\right)\right]^{-1}$. Since the Kondo screening is quenched at $T^{*}>T_{K}$, we remain in a weak Kondo screening regime even in the LL limit at $T \rightarrow 0$. The screening results in reduction of LL sound velocity, $\hbar v=\tilde{I} a_{z}$. As to the in-plane charge excitations, the formation of Kondo clouds is quenched at $T \gg T_{K}$, so instead of coherent Fermi liquid regime, $\left\langle\phi^{+} \phi^{-}\right\rangle_{\omega \rightarrow 0}$ behaves as a relaxation mode $\sim[-i \omega / \Gamma+$ $\left.\alpha q^{2}+\ln \left(\bar{\Delta} / T_{K}\right)\right]^{-1}$, where $\Gamma, \alpha$ are numerical constants.

These features of two-component electron/spin liquid manifest themselves in thermodynamics. The logarithmic corrections $\sim \ln ^{-1}\left(T^{*} / T\right)$ are expected in low- $T$ Pauli-like susceptibility of isotropic spin chains, whereas the logarithmic corrections to the susceptibility of charged layers are quenched as $\ln \bar{\Delta} / T_{K}$. The overdamped relaxation mode should be seen as a quasielastic peak in $\chi_{0}$. The $1 \mathrm{D}$ spinons contribute to the linear- $T$ term in specific heat thus mimicking the heavy-fermion behavior, while the contribution of Kondo clouds is frozen at low $T$.

In real anisotropic crystals one may expect formation of distorted and dangling chains (Fig. 3 3 ) instead of an "ideal" lattice (Fig. 1). Distortion means shift of two neighboring Kondo "shadows" in a stack. This effect may be modelled by a random overlap factor $w_{j}$ in RKKY integrals, $I_{j}=w_{j} I$. The dangling bond effect means $w_{j}=0$. Bond disorder may be treated in terms of random AFM chains [17]. According to this theory the disorder results in transformation of singlet RVB liquid into a random-singlet RVB phase with arbitrarily long singlet bonds. In chains with broken bonds the gaps arise due to the finite length effect, so the short chain segments does not contribute into the low- $\mathrm{T}$ thermodynamics. With increasing impurity concentration, the Kondo clouds be- gin to overlap and two-leg ladders with diagonal bonds arise along with isolated chains (Fig. Bb). The Nozieres' exhaustion is still not actual for these clusters. With further increase of the concentration of magnetic sites, the Doniach's problem restores in its full glory. In case of FM coupling $I$, true long-range order emerges in spin chains, but the Nozieres' exhaustion is still quenched.

One may point out the class of layered conducting/magnetic hybrid molecular solids as an object for application of above theory. These crystals are formed by alternating metallic cationic layers and insulating magnetic anionic layers with radicals $\left[\mathrm{N}(\mathrm{CN})_{2}\right]^{-}$as building blocks and transition metal ions as carriers of localized spins [18]. Organic cations with magnetic ions in such systems form ordered stacks. The problem is in preparing metallic layers with large enough Fermi surface to make Kondo screening effective and to find insulating networks with large enough distance between magnetic ions. It is worth noting that the above mentioned dicyanamide radicals with Mn ions form planar Kagome sublattice, thus being a promising object for realization of fractionalized Fermi liquid scenario proposed in [11].

This work is supported by SFB-410 project, ISF grant, A. Einstein Minerva Center and the Transnational Access program \# RITA-CT-2003-506095 at Weizmann Institute of Sciences.

[1] P. Nozieres, Ann. Phys. (Paris) 10, 19 (1985);

[2] A.M. Tsvelik ands P.B. Wiegmann, Adv. Phys. 32, 453 (1983); N. Andrei, K. Furuya, and J.H. Lowenstein, Rev. Mod. Phys. 55, 331 (1983)

[3] A.C. Hewson, The Kondo effect to Heavy Fermions (Cambridge University Press, Cambridge, 1993)

[4] J.Flouquet, in Progress in low temperature physics, (Ed. B.Halperin, Elsevier, 2005)

[5] P. Coleman, N. Andrei, J. Phys.: Cond. Mat. 1, 4057 (1989).

[6] P. Nozieres, Eur. Phys. J. B6, 447 (1998).

[7] S. Doniach, Physica B 91, 231 (1977).

[8] K.A. Kikoin, M.N. Kiselev and A.S. Mishchenko, JETP Letters, 60, 600 (1994)

[9] M.Kiselev, K. Kikoin and R. Oppermann, Phys. Rev. B 65, $184410(2002)$

[10] K.A. Kikoin, J. Phys.: Cond. Mat. 8, 3601 (1996)

[11] T. Senthil, S. Sachdev, and M. Vojta, Phys. Rev. Lett. 90, 216403 (2003) T. Senthil, M. Vojta, and S. Sachdev, Phys. Rev. B 69, 035111 (2004)

[12] N. Read and D.M. Newns, J. Phys. C: Solid State 16, 3273 (1983).

[13] L.B. Ioffe and A.I. Larkin, Phys. Rev. B 39, 8988 (1989); P.A. Lee and N. Nagaosa, Phys. Rev. B 46, 5621 (1992).

[14] V.N. Popov and S.A. Fedotov, Sov. Phys. - JETP 67, 535 (1988), M.N. Kiselev and R. Oppermann, Phys. Rev. Lett. 85, 5631 (2000).

[15] T. Giamarchi, Quantum Physics in One Dimension (Clarendon Press, Oxford, 2004).

[16] S. Eggert, I. Affleck, and M. Takahashi, Phys. Rev. Lett. 73, 332 (1994). 
[17] D.S. Fisher, Phys. Rev. B 50, 3799 (1994).

(Paris) (IV), 114, 475 (2004)

[18] J.A. Schlueter, U. Geiser, and J.L. Manson, J. Phys. 